\title{
The Effects of Changes in Family Social Capital on Health in Taiwan: An Examination of the Mediation Effect of Smoking Behavior
}

\author{
Yenhau Pan ${ }^{1}$ and Nientsu Wang, ${ }^{2, *}$ \\ ${ }^{1}$ College of Law, Minjiang University, Fuzhou, Fujian 350108, China \\ ${ }^{2}$ College of Humanities, Minjiang University, Fuzhou, Fujian 350108, China \\ "Corresponding author. E-mail: alexpan0911@163.com
}

\begin{abstract}
This study assesses the direct effect of changes in family social capital (FSC) on self-rated health (SRH) and its indirect effect through changes in smoking behavior. This study uses logistic regression and mediation analysis models to analyze the longitudinal data on 2,108 adult children in Taiwan. The findings show that respondents who maintain good relations with parents have a lower likelihood of poor SRH. The results find stable smoking exerting a detrimental effect on health. There is no evidence for the mediation effect of changes in smoking on the relationship between changes in FSC and SRH because of most of the insignificant relationships between changes in FSC and smoking. Health policy makers aiming to promote health and reduce the prevalence of smoking should understand the importance of a reciprocal relationship and the weak effects of FSC on smoking.

Keywords: Taiwan; Family social capital; Self-rated health; Smoking; Panel Study of Family Dynamics
\end{abstract}

\section{INTRODUCTION}

Previous studies indicated worse self-rate health (SRH) found in Western countries [1][2]. The increase in the proportion of reporting poor SRH is also observed in Taiwan [3]. Given that the positive association between SRH and individual-level social capital such as community participation and social trust within health literature [4][5][6][7], social capital may ameliorate the prevalence of worse SRH. To date, little attention has been paid to the importance of family social capital (FSC). More research concerning the relationship between FSC and health, as well as the mechanism through which social capital affects health is needed [8].

Coleman argues that individual-level FSC which is embedded in the structure of relationships and constitutes resources accessible to individuals can be divided into three forms: reciprocity, information channel, and effective sanction [9]. Reciprocity denotes situations where people do something for someone, who in turn feels obligated to reciprocate repay the favor someday. Information channels means information acquired through interpersonal relations. Multiple social contacts increase the frequency of health information exchange. Effective sanctions refer to informal social control to prohibit or encourage certain behaviors. Previous study has shown that reciprocity is of predictive of the quality of parent-child relationships [10]. Therefore, FSC is measured in terms of relation quality, social control and interaction which are similar to Morrow's concepts [11]. Among these forms, results have been consistently reported to show having a good relationship with parents and frequently interacting with family members positively associated with good health [12][13]. However, the effects of parental surveillance and control seem to vary depending on the measured index of health [14][15]. Despite a body of literature that supports a positive relationship between social capital and health, most studies are cross-sectional, which makes it hard to rule out the likelihood of reverse causality. As such, this study uses longitudinal data to clarify the temporal relationship between FSC and health.

Research exploring how FSC influences health is rare. A common hypothesis is that social capital enhances or reduces health behaviors, which in turn promote health [5] [16]. Poortinga [5] tested the hypothesis on the mediating role of health behaviors and indicates that social capital (e.g., civic participation and social trust) is linked to SRH, as well as to various health behaviors. Yet there is limited evidence to support the hypothesis that health behaviors mediate social capital and health. Given that the prior study tested neither the effects of FSC nor the significance of health-behavior mediation effects, this study is the first to address the issue by analyzing panel data.

There are three reasons to consider smoking as a mediator and its mediation effect may be manifested in this study. First, compared with daily alcohol intake and daily consumption of five portions of fruit and vegetable, the relationship between social capital and smoking is the strongest [5]. Next, the strong association between smoking and SRH is presented among the general population [5], rather than among older adults [17]. This current study, which uses data on general adults, may link $\mathrm{SRH}$ to smoking. Finally, SRH is a valid predictor of morbidity [18], and smoking is a major worldwide cause of mortality. Approximately 6 million people died of tobacco-related diseases per year worldwide while more 
than 20,000 Taiwanese died of tobacco use [19]. Moreover, the prevalence of smoking $(47.7 \%)$ was higher than alcohol consumption $(31.2 \%)$ and betel nut chewing $(10.4 \%)$ among Taiwanese workers [20]. To reduce the prevalence of smoking, analyzing which forms of family social capital related to smoking is needed. In a nutshell, this study aims to assess (i) whether changes in social capital influence SRH and (ii) whether changes in smoking mediate the relationship between changes in FSC and SRH, after taking into account other well-known covariates.

\section{METHOD}

\subsection{Survey Data}

This study used three waves of longitudinal data from Panel Study of Family Dynamics (PSFD) which employed a three-stage random sampling to collect data. Respondents who participated in 2005 (T1) and returned for the 4-year follow-up in 2009 (T2) provided the panel data. To test a temporal association, information on dependent variable in 2010 (T3) was collected from the same participants. At T1, 2,880 representative samples were surveyed (response rate $=74.4 \%$ ), while and 2,269 and 2,219 individuals were successfully interviewed at T2 and T3, respectively (retention rate: 79\% in 2009 and $77 \%$ in 2010). The sampling, face-to-face survey and informed consent procedure were carried out by Academia Sinica of Taiwan.

\subsection{Measures}

\subsubsection{Family social capital measures}

Three variables were based on Coleman's concepts [9] and previous studies [12][13][15]. Relation quality measured the extent of individuals getting along with their mothers and fathers. The two items were based on a five-point scale. Social interaction assessed how often respondents joined in four types of social activities. Respondents had to decide on a scale from 0 ('never') to 2 ('often'). Parental control was evaluated by asking about the degree to which mothers or fathers exerted influence on respondents' learning and entering a higher school. Respondents were asked to respond a five-point scale. Results from the factor analysis showed three distinct factors that accounted for $63.5 \%$ and $64.4 \%$ of the item variance at $\mathrm{T} 1$ and $\mathrm{T} 2$, respectively. Subjects' answering with 'well' and 'very well' for both mothers and fathers was coded as 1 , indicating good relations with their parents, while 'fair' to 'very poor' was coded as 0 , representing not getting well with their parents. The response to parental control options, 'influential' and 'most influential' were coded as 1 , denoting high levels of control, while other response options referred to low or medium levels of control.
Changes in relation quality included four categories: (I) keeping a good relationship; (ii) an 'improved relationship' (changes from not getting along with parents at T1 to getting along at T2); (iii) a 'deteriorated relationship' (changes from getting along with parents at $\mathrm{T} 1$ to not getting along at T2); and (iv) maintaining poor relations.

Four types of changes in parental control contained: (i) remaining at a high level; (ii) positive change; (iii) negative change; and (iv) remaining at a low/medium level. Changes in social interaction was assessed by subtracting $\mathrm{T} 1$ interaction summed scores from $\mathrm{T} 2$ interaction summed scores.

\subsubsection{Smoking behavior measures}

Smoking status assessed whether respondents identified themselves as current smokers, which was a valid indicator of self-reported smoking in large population studies [21]. Respondents were labeled as 1 for current smokers, i.e., they used tobacco at the time of the interview. Respondents who chose other options (e.g., an ex-smoker or someone who had never smoked) were labeled non-current smokers. Changes in smoking between 2005 and 2009 were categorized into four types: (i) stable non-smoking; (ii) taken up smoking; (iii) smoking cessation; and (iv) stable smoking.

\subsubsection{Self-rated health measurement}

SRH measured how respondents perceive current health. SRH was dichotomized into 'good' (good, excellent) and 'poor' (fair, poor, very poor). Good health was coded as 0 , whereas poor health was coded as 1 .

\subsubsection{Control variables}

Control variables include age, education, employment, family structure, house moving, and chronic disease (see Table 1).

\subsection{Data Analysis}

The current study applied an expectation maximization (EM) algorithm to impute the missing values of FSC via SPSS 22.0 due to more accurate estimates of EM than those of conventional methods. A total of 2,108 respondents were included the final analysis. The imputed result was highly similar to original one deleting missing values.

A logistic regression model was employed to analyze whether changes in social capital and smoking were associated with SRH over time. Model 1 included changes in relationship quality, social interaction parental control, and baseline SRH and control variables. Changes in smoking were added to Model 2. To address the second research question, this study used a mediation analysis 
method (referred to as the KHB method), developed by Karlson, Holm, \& Breen [22], to test the significance of mediation effects.

\section{RESULTS}

Table 1 shows the descriptive statistics of changes in FSC, smoking, SRH and covariates at baseline. The proportion with regard to current smoking decreased from $27.2 \%$ to $25.1 \%$ between 2005 and 2009. The proportion of respondents who had poor SRH increased from $56.9 \%$ to $62.8 \%$ over a 5 -year period.

Table 2 analyzes the effects of changes in FSC and smoking on health. When adjusting for baseline SRH and covariates, respondents who stated they had a deteriorated relationship with parents or maintained a good relationship with parents were less likely to report poor SRH than those who maintained a poor relationship with parents (OR of 0.68 and OR of 0.65 , respectively; Model 1). In Model 2, a stable smoker had significantly higher ORs for reporting poor health than a stable non-smoker. When a change in smoking behavior is included in Model 2 , the effect of having a deteriorated relationship with parents becomes insignificant.

Table 1. Descriptive statistics of variables used in this study

\begin{tabular}{|c|c|c|c|}
\hline Characteristics & T1(2005) & T2(2009) & T3(2010) \\
\hline \multicolumn{4}{|l|}{ Relation quality(N, \%) } \\
\hline $\begin{array}{l}\text { Not getting well with } \\
\text { parents }\end{array}$ & $524(24.9)^{*}$ & $579(27.5)$ & \\
\hline Getting well with parents & $1584(75.1)$ & $1529(72.5)$ & \\
\hline Social interaction(M, SD) & $6.58(1.53) *$ & $6.35(1.59)$ & \\
\hline \multicolumn{4}{|l|}{$\begin{array}{l}\text { Parental control } \\
(\mathrm{N}, \%)\end{array}$} \\
\hline Low or medium level & $1550(73.5)$ & $1513(71.8)$ & \\
\hline High level & $558(26.5)$ & $595(28.2)$ & \\
\hline \multicolumn{4}{|l|}{ Smoking } \\
\hline $\begin{array}{l}\text { Current non-smoker(N, } \\
\%)\end{array}$ & $1534(72.8)^{*}$ & $1579(74.9)$ & \\
\hline Current smoker & $574(27.2)$ & $529(25.1)$ & \\
\hline \multicolumn{4}{|l|}{$\begin{array}{l}\text { Self-rated health } \\
(\mathrm{N}, \%)\end{array}$} \\
\hline Poor health & $\begin{array}{l}1200 \\
(56.9)^{* * *}\end{array}$ & & $1324(62.8)$ \\
\hline Good health & $908(43.1)$ & & $784(37.2)$ \\
\hline \multicolumn{4}{|l|}{ Gender(N, \%) } \\
\hline Male & $1048(49.7)$ & & \\
\hline Female & $1060(50.3)$ & & \\
\hline \multicolumn{4}{|l|}{ Age $(\mathrm{N}, \%)$} \\
\hline $29-44$ & $729(34.6)$ & & \\
\hline $45-60$ & $840(39.8)$ & & \\
\hline $60+$ & $539(25.6)$ & & \\
\hline \multicolumn{4}{|l|}{ Education (N, \%) } \\
\hline Basic education & $815(38.7)$ & & \\
\hline Middle education & $798(37.9)$ & & \\
\hline Higher education & $495(23.4)$ & & \\
\hline \multicolumn{4}{|l|}{ Job status(N, \%) } \\
\hline Unemployment & $635(30.1)$ & & \\
\hline Employment & $1473(69.9)$ & & \\
\hline \multicolumn{4}{|l|}{$\begin{array}{l}\text { Family structure } \\
(\mathrm{N}, \%)\end{array}$} \\
\hline Non-intact family & $403(19.1)$ & & \\
\hline intact family & $1705(80.9)$ & & \\
\hline \multicolumn{4}{|l|}{$\begin{array}{l}\text { House moving } \\
(\mathrm{N}, \%)\end{array}$} \\
\hline House non-mover & 2033 (96.4) & & \\
\hline House mover & $75(3.6)$ & & \\
\hline $\begin{array}{l}\text { Chronic disease } \\
(\mathrm{N}, \%)\end{array}$ & & & \\
\hline
\end{tabular}

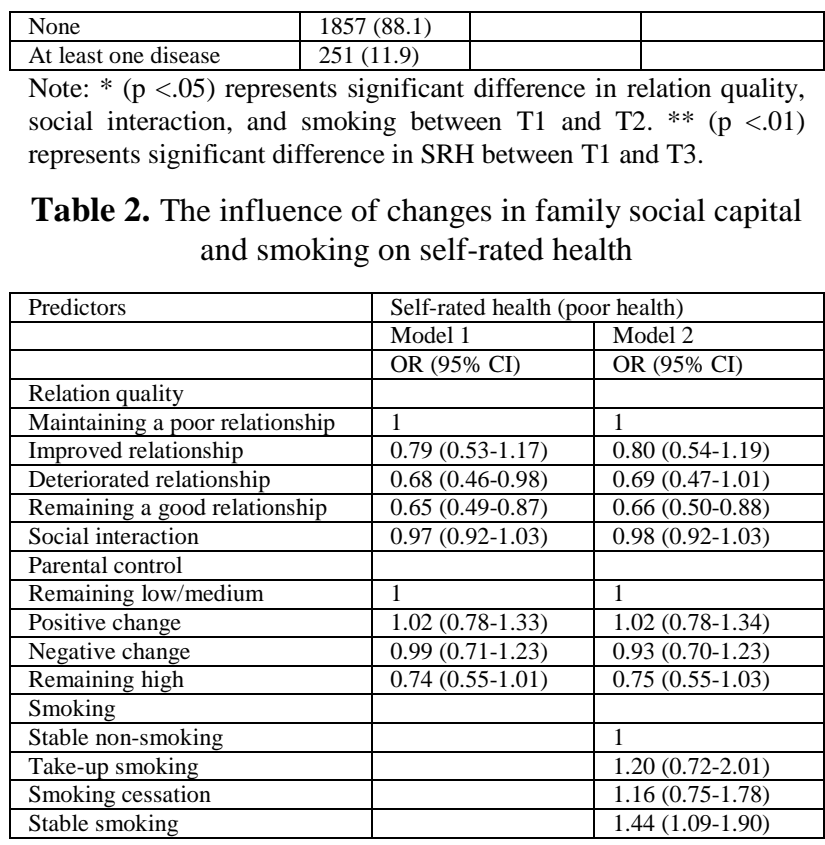

Note. Models 1 and Model 2 adjusted for covariates.

\section{DISCUSSION}

This study uses longitudinal data sets to analyze the effect of changes in FSC on SRH and how changes in FSC affect health through changes in smoking behavior. Although most cross-sectional studies view FSC as a crucial factor protecting against health problems [12] [13] [15], this study reveals that only maintaining a good relationship with parents benefits health. Prior to including changes in smoking behavior, the finding that a deteriorated relationship with parents lowers the likelihood of reporting poor health is unanticipated. A possible explanation could be that, as the deteriorated relationship is due to imbalance in reciprocity, every adult child giving more support to their parent reduces the extent of the caregiving burden [23], which, in turn, is associated with good health [24]. Filial piety, one of the relevant values in modern Chinese societies, makes it possible for any family member to provide elderly parents with more instrumental support and emotional care. However, the weak effect of a deteriorated relationship disappears in the final model.

The result that changes in social interaction are not related to SRH differed from that of the prior work, which found that fewer social interactions were associated with poor health in students in England [12]. Previous research, relying on cross-sectional design, cannot make casual claims. Perhaps a healthy respondent is more likely to engage in a variety of activities within family. In accordance with evidence from cross-sectional research [12][13], the non-significant impact of control on SRH is found in this study. The results suggest that quality of the relationship plays a more important role in promoting health than two other forms of social resources. The findings not only deepen the understanding of the health protection effects of relation quality based on Coleman [9], 
but also extend the scope from the influence of relation quality on elder parents' wellbeing in Western countries [25] to on adult children' health in a non-Western country. A good relationship with parents represents a reciprocal relationship in which respondents are easily exposed to instrumental and emotional resources that promote health. The detrimental effect of smoking on health is as stated by these studies[5][26]. However, the finding that stable non-smoking improves SRH is inconsistent with the longitudinal study conducted showing that stable non-smoking does not affect baseline SRH, compared with stable smoking [26]. It is difficult to assess the effect of smoking behavior changes on health because of smoking and health measurements being conducted at the same time in their study.

The KHB mediation analysis shows that none of the indirect effects is significant. The non-significant indirect effect of changes in social capital, via smoking behavior changes, on SRH can be attributed to most of the insignificant relationships between changes in social capital and smoking. Different social meanings of tobacco, which vary at different life stages, could dilute the effect of relationship quality. For some adults, social smoking takes place because tobacco is sometimes viewed as a means of socializing with important others [27].

This study confirms that smoking does not mediate the relationship between FSC and SRH, which is similar to earlier research focusing on other forms of social capital [5]. Future research should further explore whether family-related social capital promote health through health-promoting behaviors such as eating a low-fat diet and regular exercise.

These findings are subject to several limitations. First, while the PSFD sample was selected to reflect the Taiwanese adult population, those aged below 28 were not included in the survey population. Therefore, the results can only be applied to adults aged over 29 years, rather than all adults aged over 18 years. Furthermore, this study does not consider other forms of FSC, including emotional support, instrumental support and family network, as categorized by Alvarez et al. [8]. Failure to include these variables in the analytic model may influence the estimates of the relation quality effect.

\section{CONCLUSION}

The results identify that, of the three forms of FSC, only maintaining a harmonious relationship with parents is beneficial for improving adult children's SRH. Stable smoking appears to result in poor health. Besides exploring Putnam's concept defining social capital as features of social organization, future researchers should pay more attention to the effects of FSC. This study finds most of family-related social capital measures failing to affect smoking. Thus, no mediation effect of changes in smoking on the association between changes in FSC and SRH is found. Health policy makers aiming to promote health and reduce the prevalence of smoking should understand the importance of a reciprocal relationship and the weak effects of FSC on smoking in an adult population as well as the social meaning of tobacco use in a given context.

\section{REFERENCES}

[1] M. Eriksson, and N. Ng., "Changes in access to structural social capital and its influence on self-rated health over time for middle-aged men and women: A longitudinal study from northern Sweden", Social Science and Medicine, vol. 130, 2015, pp.250-258.

[2] R. Fenwick, and M. Barresi, C., "Health consequences of marital-status change among the elderly: A comparison of cross-sectional and longitudinal analyses", Journal of Health Social Behavior, vol.22, 1981, pp.106-116.

[3] F.H. Jhang, "Changes in marital status and links to self-rated health: A study of temporal relationships", social science journal, vol.55, 2018, pp.87-96.

[4] T. Meng, and H. Chen, "A multilevel analysis of social capital and self-rated health: Evidence from China. Health and Place", vol.27, 2014, pp.38-44.

[5] W. Poortinga, "Do health behaviors mediate the association between social capital and health?" Preventive Medicine, vol.43, 2006a, pp.488-493.

[6] W. Poortinga, "Social relations or social capital? Individual and community health effect $\mathrm{s}$ of bonding social capital. Social Science and Medicine”, vol.63, 2006b, pp.255-270.

[7] J. W. Snelgrove, H. Pikhart, and M. Stafford, “A multilevel analysis of social capital and self-rated health: Evidence from the British Household Panel Survey”. Social Science and Medicine, vol.68, 2009, pp.1993-2001.

[8] E. C. Alvarez, I. Kawachi, and J. R. Romani, "Family social capital and health - a systematic review and redirection". Sociology of Health and Illness, vol.39, 2017, pp.5-29.

[9] J. S. Coleman, "Social capital in the creation of human capital". American Journal of Sociology, vol.94, 1988, pp.95-120.

[10] B. Schwarz, G. Trommsdorff, I. Albert, and B. Mayer, "Adult parent-child relationships: Relationship quality, support, and reciprocity". Applied Psychology: An International Review, vol.54, 2005, pp.396-417.

[11] V. Morrow, "Conceptualizing social capital in relation to the well-being of children and young people: A critical review". The Sociological Review, vol.44, 1999, pp.744-765.

[12] A. Morgan, and B. J. A. Haglund, "Social capital does matter for adolescent health: Evidence from the 
English HBSC study". Health Promotion International, vol.24, 2009, pp.363-372.

[13] D. Nowak, E. Suzuki, and I. Kawachi, “Are family, neighborhood and school social capital associated with higher selfrated health among Croatian high school students? A population-based study". 2015, BMJ Open,5, doi:10.1136/ bmjopen-2014-007184.

[14] A. Morgan, F. Rivera, C. Moreno, and B.J. Haglund, "Does social capital travel? Influences on the life satisfaction of young people living in England and Spain”. BMC Public Health, vol.12, 2012, doi: 10.1186/1471-2458-12-138.

[15] C. Rothon, L. Goodwin, and S. Stansfeld, "Family social support, community "social capital" and adolescents' mental health and educational outcomes: A longitudinal study in England". Social Psychiatry and Psychiatric Epidemiology, vol.47, 2012, pp.697-709.

[16] J. Schultz, A. M. O'Brien, and B. Tadesse, "Social capital and self-rated health: Results from the US 2006 social capital survey of one community". Social Science and Medicine, vol.67, 2008, pp.606-617.

[17] L. Abuladze, N. Kunder, K. Lang, and S. Vaask, "Associations between self-rated health and health behaviour among older adults in Estonia: A crosssectional analysis". BMJ Open, 7, 2017, doi:10.1136/bmjopen-2016-013257.

[18] E. L. Idler, and Y. Benyamini, "Self-rated health and mortality: a review of twenty-seven community studies". Journal of Health and Social Behavior, vol.38, 1997, pp.21-37.

[19] Ministry of Health and Welfare. "Adult smoking behavior survey". (2016). Available at https://www.hpa.gov.tw/EngPages/Detail.aspx?nodeid $=1077 \&$ pid=6202 (accessed 20 January 2018).

[20] W. M. Liang, H. M. Kuo, and C. B. Wang, "Prevalence of tobacco smoking, drinking and betel nut chewing among Taiwanese workers in 1999". Mid-Taiwan Journal of Medicine, vol.7, 2002, pp.146-154.

[21] M. Rebagliato, "Validation of self-reported smoking". Journal of Epidemiology and Community Health, vol.56, 2002, pp.163-164.

[22] K. B. Karlson, A. Holm, and R. Breen, "Comparing regression coefficients between same-sample nested models using logit and probit: A new method". Sociological Methodology, vol.42, 2012, pp.286-313.

[23] D. W. Lai, "Cultural predictors of caregiving burden of Chinese-Canadian family caregivers". Canadian Journal on Aging, vol.26, 2007, pp.15-26.

[24] I. Abdollahpour, S. Nedjat, M. Noroozian, Y. Salimi, and R. Majdzadeh, "Caregiver burden: The strongest predictor of self-rated health in caregivers of patients with dementia". Journal of Geriatric Psychiatry and Neurology, vol.27, 2014, Pp.172-180.

[25] L. G. Bell, and D. C. Bell, "Positive relationships that support elder health and well-being are grounded in midlife/adolescent family". Fam Community Health, vol.35, 2012, pp.276-286.

[26] K. Sargent-Cox, N. Cherbuin, L. Morris, P. Butterworth, and K. J. Anstey," The effect of health behavior change on self-rated health across the adult life course: A longitudinal cohort study". Preventive Medicine, vol.58, 2014, pp.75-80.

[27] F. N. Hsia, D. Spruijt-Metz, "Acculturation and meanings of smoking among Asian-American college students". Addictive Behaviors, vol.32, 2007, pp.2292-2296. 